\title{
Do Neural Factors Underlie Age Differences in Rapid Ankle Torque Development?
}

\author{
Darryl G. Thelen, PhD, ${ }^{* \neq}$ James A. Ashton-Miller, PhD, ${ }^{* \neq}$ Albert B. Schultz, PhD, ${ }^{* \neq}$ and \\ Neil B. Alexander, $M D^{\dagger \neq}$
}

\begin{abstract}
OBJECTIVES: Rapid torque development is substantially slower in healthy old adults compared with young adults, but the underlying cause of this age-related loss remains unclear. Measurements of myoelectric signals in ankle dorsi- and plantarflexor muscles during rapid exertions were used to explore the extent to which the loss might be attributed to neural factors.
\end{abstract}

METHODS: Myoelectric signals were measured in a laboratory setting in 24 healthy young and 24 healthy old adult volunteers during rapid isometric and isokinetic torque development. Premotor times, muscle activation rates, and myoelectric activity levels of agonistic and antagonistic muscles were quantified.

RESULTS: There were few marked age differences in the premotor times or in the onset rates or magnitudes of agonistic muscles activities during maximum isometric and during isokinetic exertions. Premotor times were statistically associated with age but, in the mean, were only approximately 10 to $25 \mathrm{~ms}$ longer in the old. Age effects on agonist muscle activity magnitudes were significant only in the lateral gastrocnemius. Small decreases in antagonistic muscle activity levels with age were found.

CONCLUSIONS: Given the outcomes of this study, the differences observed previously in rapid torque development abilities in healthy older adults, compared with healthy younger adults, seem attributable largely to differences in muscle contraction mechanisms rather than to differences in speeds of stimulus sensing or central processing of motor commands, or to differences in muscle recruitment strategies. J Am Geriatr Soc 44:804-808, 1996.

\footnotetext{
From the "Department of Mechanical Engineering and Applied Mechanics, tDivision of Geriatric Medicine and VA Medical Center GRFCC, and \#Institute of (icrontology, University of Michigan, Ann Arbor, Michigan.

Supported by NIH grants AG06621, AG08808, and AG10542.

Address correspondence to Albert B. Schultz, PhD, Biomechanics Research Laboratory, Department of Mechanical Engineering and Applied Mechanics, University of Michigan, Ann Arbor, MI 48109-2125.
}

Substantial declines in dynamic strength capabilities are

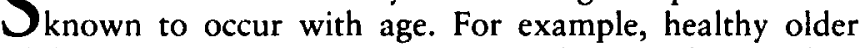
adults $(\mathrm{OA})$ can require approximately $50 \%$ longer than younger adults (YA) to develop moderate to large ankle torques and demonstrate strength losses of up to $40 \%$ during rapid isometric and during isokinetic exertions (Thelen et al. $\left.{ }^{1}\right)$. The underlying causes of these age losses in dynamic strength remain unclear. They may be attributable to neural factors: age-related delays in sensing environmental stimuli; delays in processing those stimuli and issuing to the muscles the neural signals commanding appropriate responses; or age-related changes in strategies for muscle recruitment. Alternately, these losses might be attributable to age-related changes in muscle contraction mechanics. ${ }^{2}$

Myoelectric signal (MES) measurements provide one means to assess possible sources of age-related losses in dynamic strengths. During isokinetic exertions, mean muscle activity levels of agonistic muscles have been found to be independent of contraction velocity ${ }^{3,4}$ and age, ${ }^{5}$ with activity levels of antagonistic muscles found to be relatively small, ranging from 2 to $20 \%$ of maximum activites. ${ }^{6}$ These observations suggest that factors related to muscle contraction mechanics, rather than neural factors, may underlie agerelated loss in isokinetic strength. ${ }^{5}$ However, rates of muscular activation and the effects of age on muscle activity levels during rapid isometric strength development have not been well explored.

The objective of the present study was to quantify myoelectric activation rates and recruitment patterns of ankle dorsi- and plantarflexor muscles in healthy young and old adults during rapid maximum isometric and isokinetic exertions. In order to assess the contributions of neural factors to age-related losses in dynamic strength, we tested null hypotheses that neither age, gender, nor joint rotation velocity affects muscle premotor times, rates of muscle activation, or the magnitudes of agonistic and antagonistic muscle activities.

Knowledge of likely sources of age-related declines in dynamic strengths is bound to allow for earlier and improved assessment of impending disability and enable the design of more effective interventions and better direct research into mobility impairments among older people.

\section{METHODS}

\section{Subjects}

Twenty-four healthy young (YA, ages 19-29 years) and 24 healthy old (OA, ages 65-86 years) adults, each group 
containing 12 females (YF, OF) and 12 males (YM, OM), participated in this study. YA were significantly $(P<.05)$ taller than OA (means for YF, YM, OF, and OM were 165.3, $177.0,158.9$, and $174.3 \mathrm{~cm}$, respectively) whereas body masses were not significantly different between age groups (mean masses for YF, YM, OF, and OM were 59.4, 72.9, 60.0 , and $74.5 \mathrm{~kg}$ ). OA were all independent, communitydwelling residents who were recruited from social and recreational programs sponsored by a University-affiliated geriatrics clinic or from among volunteers participating in the Claude Pepper Geriatric Research and Training Center $\mathrm{Hu}$ man Subjects Core. YA were recruited by advertisement among University staff and students.

OA underwent a standardized medical history and physical examination that focused on neurological ${ }^{7}$ and musculoskeleta ${ }^{8}$ findings. These, which are detailed by Alexander et al., ${ }^{9}$ were conducted by a nurse clinician under the supervision of a physician geriatrician. Although OA denied significant neurological, musculoskeletal, or otological abnormalities, on examination 11 subjects had experienced rare, occasional pain in the lower extremities or lower back but were presently not symptomatic; 10 subjects had decreased lower extremity reflexes; two subjects had reduced vibration sens $\epsilon$ at the medial malleolus; and one subject had decreased position sense at the great toe. Such findings upon examination are common in otherwise healthy adults. ${ }^{10}$ In fact, these OA likely represented a highly capable cohort in that all except one $O A$ were physically active and participated regularly in walking, exercise, or sports activities.

\section{Tasks and Measurements}

Subjects, using the foot they said they would use to kick a ball, made both rapid isometric and isokinetic exertions against an isokinetic dynamometer (MERAC, Universal Gym Equipment) instrumented specifically for this study. Subjects were tested supine and had ample body segment support as detailed by Thelen et al. ${ }^{1}$

In all tasks, subjects were asked to "push down on" (Plantarflexion or PF) or "pull up on" (Dorsiflexion or DF) a footplate "as fast and as hard as possible" in response to a visual cue. The cue was provided by a flash lamp that was located approximately 1 meter directly above the subject's eyes and programmed to illuminate after a random delay of 1 to 2 seconds following an aural warning cue. During isometric tasks, the footplate was held at a preset angle, and subjects were asked to push or pull on the plate maximally until hearing a tone that sounded 3 seconds after the flash. Isokinetic exertions started from a position of extreme DF and PF during PF and DF exertions, respectively, so that exertions could reach maximum levels by the time of passing through more comfortable positions. Subjects were asked to push or pull throughout their available range of ankle motion. Isokinetic exertions were performed at nominal angular velocities of $30,60,120,180$, and $240 \mathrm{deg} / \mathrm{s}$. Subjects were strongly encouraged to give maximum efforts, with visual feedback provided on the torque levels attained.

Subjects made 30 exertions in PF followed by 30 exertions in DF. Twelve initial practice trials, consisting of six submaximum and six maximum exertions, enabled subjects to become familiar with the tasks. The 18 trials in each of PF and DF from which data were collected were then performed in identical blocks of six trials each. In each block, one exertion was made isometrically, and one exertion was made at each of the five isokinetic nominal speeds, in the same initially randomized, fixed trial sequence. Subjects rested approximately 30 seconds between trials and 5 minutes between blocks.

Surface myoelectric signals (MES) were recorded over the mid-bellies of tibialis anterior (TA), soleus (SOL), and medial (MGAS) and lateral (LGAS) gastrocnemius muscles using bipolar surface silver disc electrodes with $2-\mathrm{cm}$ centerto-center spacing. Electrodes were filled with gel and placed parallel with the underlying muscle fiber direction.

\section{Data Analysis}

Myoelectric signals were amplified to volt levels using a differential amplifier with a $30-\mathrm{Hz}$ high-pass filter to attenuate movement artifacts. MES were digitized at $1.4 \mathrm{kHz}$ using a 12-bit A/D board. Root-mean-square (RMS) MES were calculated using a 25 -ms center-average moving window. RMS MES were then low-pass filtered at $30 \mathrm{~Hz}$, using a 6th order bi-directional Butterworth filter, and resampled at 200 $\mathrm{Hz}$.

The trial-maximum absolute activity level was found for each muscle when it acted as an agonist during a maximum isometric exertion for each subject. That level was the peak RMS MES averaged over a 1-second interval. Where appropriate, processed MES were subsequently normalized to these maximum isometric activity levels.

Premotor time (PMT) was defined as the time between the light flash and the onset of agonist muscle activity. For each exertion, the time of onset of activity was calculated by first determining the baseline noise level and the just-defined maximum activity levels during the exertion, identifying the times at which 5 and $10 \%$ of the maximal activity were reached and linearly extrapolating back to the baseline noise level to estimate the onset time (Figure 1).

Trial-mean absolute activity levels were quantified as the average RMS MES, over a velocity-dependent time interval, for each muscle and for each subject. The interval started 200 ms after PMT because RMS MES typically reached steady levels by this time. For isokinetic tasks, interval length was set to the additional time beyond $200 \mathrm{~ms}$ after PMT required to rotate the ankle through $45 \mathrm{deg}$. The interval during isometric tasks was set equal to that for the $30-\mathrm{deg} / \mathrm{s}$ isokinetic task.

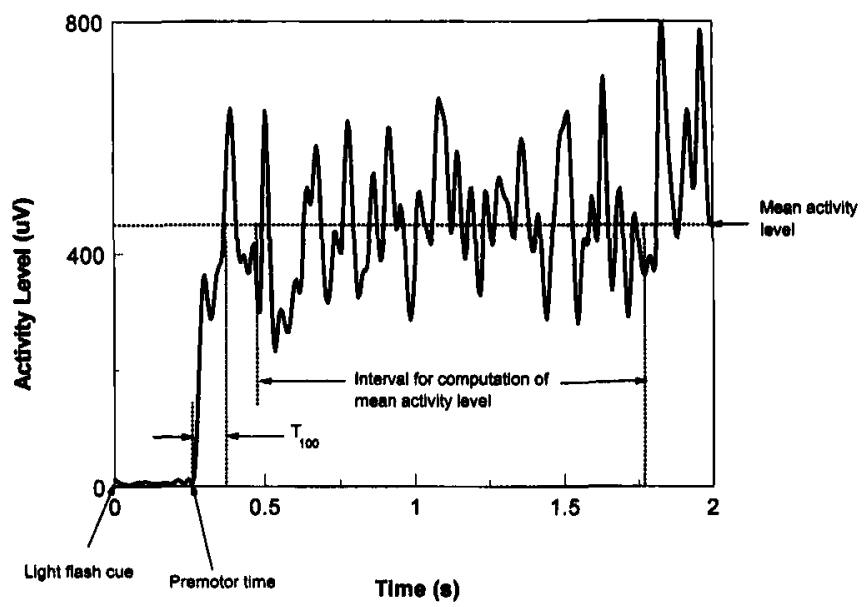

Figure 1. A representative muscle activity record (tibialis anterior myoelectric activity during isometric dorsiflexion) showing the parameters used to quantify the record. 
The rate of activation of the agonistic muscles during rapid isometric exertions was gauged by the time from PMT to the time at which $100 \%$ of trial-mean absolute MES was first reached, denoted here as $\mathrm{T}_{100}$ (Figure 1).

\section{Statistical Analyses}

For each subject, the block-mean of the variables over the three trials, one per block, was calculated first. From these block-means, group means and standard deviations were calculated for YF, OF, YM, and OM, or for YA and OA, as appropriate. A three-way analysis of variance (ANOVA) was performed to test the effect of age, gender, and muscle on PMT of muscles when acting as agonists. All other statistical analyses were performed after stratifying by muscle. Twoway ANOVA was used to test the effect of age and gender on mean normalized-MES and $T_{100}$ during isometric exertions. A three-way ANOVA was performed to test the effect of age, gender, and angular velocity on mean MES during isokinetic exertions. $P$ values $<.01$ were considered statistically significant throughout.

\section{RESULTS}

Myoelectric signal (MES) data from the nominal 180 and $240 \mathrm{deg} / \mathrm{s}$ speeds are not reported here, since many OA could not reach those prescribed velocities, especially in dorsiflexion. ${ }^{1}$ In the following, all values referred to are group-mean values.

\section{Premotor Times}

Premotor times ranged from 115 to $153 \mathrm{~ms}$ (Table 1 ). PMT were significantly dependent on age $(P<0.001)$ with OA being 9 to $27 \mathrm{~ms}$ slower than $\mathrm{YA}$ in initiating muscle activity. However PMT were independent of gender and muscle. That is, PMT of the TA dorsiflexor during DF exertions were similar to the PMT of plantarflexor muscles ( $\mathrm{SOL}$, MGAS, LGAS) during PF exertions, for both males and females.

Table 1. Effects of Age, Gender and Muscle on Group-Mean Premotor Times and Times from Premotor Time to First Reaching Mean MES Level, $T_{100}$, in Agonist Muscles During Rapid Isometric Exertions

\begin{tabular}{cccccc}
\hline Direction & Muscle & YF† & OF & YM† & OM \\
\hline Premotor Times (ms) & & & & \\
DF & TA & $115(26)$ & $142(24)$ & $137(39)$ & $142(29)$ \\
PF & MGAS & $121(32)$ & $148(36)$ & $124(17)$ & $138(21)$ \\
& LGAS & $127(29)$ & $151(39)$ & $133(20)$ & $142(24)$ \\
\multicolumn{7}{c}{ Times (ms) } & SOL & $131(32)$ & $152(39)$ & $142(22)$ & $153(26)$ \\
DF & TA & $88(23)$ & $99(46)$ & $83(47)$ & $81(53)$ \\
PF & MGAS & $86(46)$ & $98(51)$ & $90(41)$ & $97(69)$ \\
& LGAS & $90(38)$ & $84(43)$ & $92(41)$ & $72(39)$ \\
& SOL & $89(37)$ & $83(38)$ & $82(40)$ & $70(46)$ \\
\hline
\end{tabular}

Standard deviations in parentheses.

+ Significant age $(P<.001)$ effects by ANOVA.

$\ddagger$ No significant age or gender effects by ANOVA.

Subjects $Y F, O F, Y M, O M: Y=Y$ Young, $O=$ Old, $F=$ Female, $M=$ Male

Direction: $\mathrm{DF}=$ dorsiflexion and $\mathrm{PF}=$ plantarflexion.

Muscle: TA $=$ Tibialis Anterior, MGAS $=$ Medial Gastrocnemius, LGAS = l.ateral Gastrocnemius, SOI. = Soleus.

\section{Rate of Activation}

The time intervals, $T_{100}$, from PMT to first reaching trial-mean MES level ranged from 72 to $99 \mathrm{~ms}$ (Table 1). These intervals, and thus the rate of activation of agonistic muscles, were independent of age and gender (Figure 2 shows group mean data). $\mathrm{T}_{100}$ values were variable across subjects, with the coefficient of variation (SD/Mean) ranging from 0.25 to 0.70 .

\section{Agonistic Muscle Activity Levels}

During the isometric exertions, the group-mean values of the trial-mean absolute MES amplitudes ranged from 99 to $452 \mathrm{mV}$, with the amplitudes smallest in the LGAS and largest in the TA muscle (Table 2). MES amplitudes for all muscles were significantly larger in males than in females, but they were independent of age. The male-to-female ratio of activity levels ranged from 1.1 to 1.6 for YA and from 2.0 to 2.5 for OA.

During isokinetic exertions, MES levels of agonistic muscles (Table 3 ) were nearly the same as isometric activity levels for all angular velocities through $120 \mathrm{deg} / \mathrm{s}$. Age effects on agonist mean MES were not significant, except for the LGAS muscle during PF exertions, in which activity was higher in OA than in YA $(P=.002)$. There was a tendency for MES levels to increase with velocity, but this effect reached significance only for the LGAS muscle $(P<.001)$.

\section{Antagonistic Muscle Activity Levels}

During the isometric exertions, the group-mean values of the antagonistic MES amplitudes ranged from 8 to $23 \%$ of maximum agonist values (Table 3 ). During isokinetic exertions, mean MES of antagonistic muscles ranged from 9 to $48 \%$ of maximum activity levels.

OA exhibited significantly smaller antagonistic activities in the TA muscle during isokinetic PF exertions than did YA $(P=.008)$. For example, TA muscle normalized activity of YA was nearly twice that of OA during $30 \mathrm{deg} / \mathrm{s}$ PF exertions. Antagonistic muscle activities during DF exertions tended to be greater in OA than in YA, but the differences did not reach significance. Coactivation of TA during PF exertions and of both MGAS and LGAS muscles during DF exertions increased significantly with increasing angular velocity $(P<$ $.001)$

\section{DISCUSSION}

Quantification of myoelectric signals (MES) during maximum exertions was used to explore the extent to which neural factors, that is, factors involved in the processes that occur before the arrival of the MES at the muscles, might contribute to observed age-related differences in dynamic strength characteristics. The MES data showed no age-group differences in rate of muscular activation and only minor age-group differences in premotor times and magnitudes of muscular activation. These findings suggest that age-related differences in rapid isometric and isokinetic torque development do not result from differences in speeds of stimulus sensing or central processing of motor commands or from any major differences in muscle recruitment strategies. Thus, these differences are probably attributable to age-related changes in muscle contraction mechanics, that is, in at least some of the processes that occur after the arrival of the MES at the muscles. 


\section{Isometric Dorsiflexion}

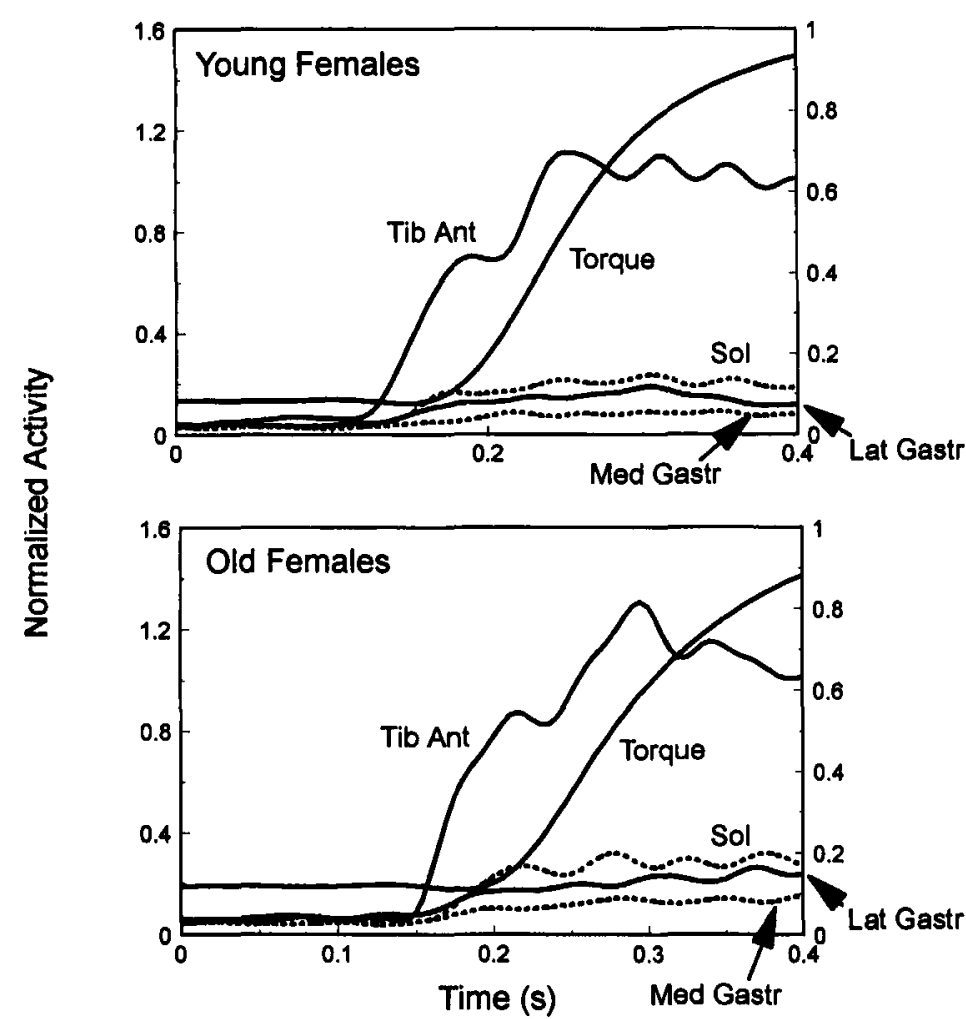

\section{Isometric Plantarflexion}
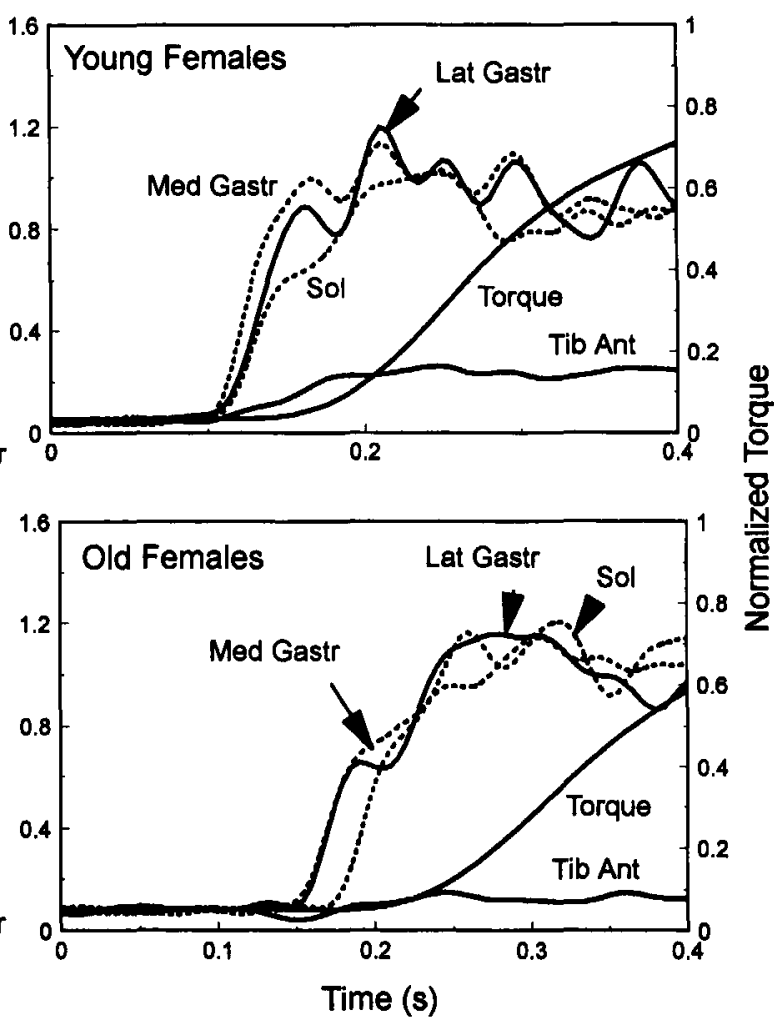

Figure 2. Mean normalized myoelectric activity and normalized torque histories among each of the four subject groups. Normalizations are to maximum isometric activity levels and torques.

Table 2. Effects of Age and Gender on Group-Mean Absolute Myoelectric Activities in Agonist Muscles During Rapid Isometric Exertions

\begin{tabular}{|c|c|c|c|c|c|}
\hline \multirow[b]{2}{*}{ Direction } & \multirow[b]{2}{*}{ Muscle } & \multicolumn{4}{|c|}{ Myoelectric Activities $(\mu V)$} \\
\hline & & YF & OF & YM & OM \\
\hline DF & TAT & $264(90)$ & 294 (133) & $323(90)$ & $452(267)$ \\
\hline \multirow[t]{3}{*}{ PF } & MGAS§ & $248(146)$ & $150(89)$ & $284(73)$ & $298(121)$ \\
\hline & LGAS $\ddagger$ & $160(66)$ & $99(53)$ & $244(81)$ & $251(156)$ \\
\hline & $\mathrm{SOL}^{*}$ & $187(82)$ & $144(59)$ & 291 (124) & $333(217)$ \\
\hline
\end{tabular}

Standard deviations in parentheses.

+ Gender $(P=020)$ effects by ANOVA.

Significant gender $(P=.007)$ effects by ANOVA

$\ddagger$ Significant gender $(P<.001)$ effects by ANOVA.

* Significant gender $(P<.001)$ effects by ANOVA.

See Table 1 for definitions of abbreviations.

Premotor times were on the order of 110 to $150 \mathrm{~ms}$, similar in value to those found in the lower extremity during visually-cued simple reaction time tasks (Ashton-Miller, Alexander, Grenler et al., manuscript submitted). The premotor times (latencies) between visual cue and muscle activity initiation were prolonged slightly in the $\mathrm{OA}$, compared with the YA, by approximately 10 to $25 \mathrm{~ms}$ or 10 to $20 \%$ percent. This is in agreement with age differences found during simple reaction time tests in both upper and lower extremities. ${ }^{11-1.5}$

The $T_{100}$ times, from PMT to first reaching trial-mean MES level, indicative of the rate of activation of agonistic muscles, were independent of age. Mean $\mathrm{T}_{100}$ times were on the order of $100 \mathrm{~ms}$ and were independent of the muscle under investigation. The variability of these measures across tasks and subjects partially results from the random variations present in surface myoelectric signals.

The age difference found with regard to muscle activity levels was that YA used greater coactivation of the TA muscle during PF exertions than did OA. This finding contrasts with observations by others ${ }^{16,17}$ that old adults tend to use more co-contraction than do young adults in another task, the maintenance of standing balance. Co-contraction is believed to be used during standing to maintain whole body stability. Perhaps because of the whole body support provided by the equipment used in the present experiments, needs for use of co-contraction were smaller than those arising in maintenance of standing balance. Solonomow et al. ${ }^{18}$ argued that cocontraction may be used during strength tests to augment the role of the ligaments in stabilizing a joint and to equalize joint contact force distributions. Perhaps these issues were of greater concern for YA than for OA subjects in the present experiments, given the larger absolute joint torques developed by the young. ${ }^{1}$

The extent to which present findings are generalizable to other subject populations or to behaviors of other muscle groups in the present population was not examined and so is not known. Thelen et al. ${ }^{1}$ document that the absolute isometric strengths of our subjects were similar in value to those reported for other subjects in other studies. We chose to use surface rather than needle electrodes for reasons of subject 
Table 3. Effects of Age and Velocity on Group-Mean Normalized Myoelectric Activity Levels

\begin{tabular}{|c|c|c|c|c|c|c|c|c|c|}
\hline \multirow[b]{2}{*}{ Direction } & \multirow[b]{2}{*}{ Muscle } & \multicolumn{2}{|c|}{ Isometric } & \multicolumn{2}{|c|}{$30 \mathrm{deg} / \mathrm{s}$} & \multicolumn{2}{|c|}{$60 \mathrm{deg} / \mathrm{s}$} & \multicolumn{2}{|c|}{$120 \mathrm{deg} / \mathrm{s}$} \\
\hline & & YA & OA & YA & OA & YA & OA & YA & OA \\
\hline \multicolumn{10}{|c|}{ Normalized Agonistic Activity Levels } \\
\hline DF & TA & $0.94(0.04)$ & $0.95(0.04)$ & $0.96(0.11)$ & $0.96(0.17)$ & $0.98(0.11)$ & $1.00(0.28)$ & $1.03(0.13)$ & $0.97(0.26)$ \\
\hline \multirow[t]{3}{*}{ PF } & MGAS & $0.89(0.09)$ & $0.86(0.10)$ & $0.93(0.33)$ & $0.97(0.23)$ & $0.95(0.30)$ & $1.02(0.23)$ & $0.91(0.27)$ & $1.14(0.33)$ \\
\hline & LGASł & $0.88(0.07)$ & $0.85(0.09)$ & $0.98(0.26)$ & $1.08(0.35)$ & $0.99(0.27)$ & $1.16(0.43)$ & $1.01(0.24)$ & $1.37(0.61)$ \\
\hline & SOL & $0.86(0.09)$ & $0.88(0.08)$ & $0.90(0.33)$ & $0.92(0.25)$ & $0.90(0.36)$ & $1.10(0.38)$ & $0.94(0.39)$ & $1.20(0.70)$ \\
\hline \multicolumn{10}{|c|}{ Normalized Antagonistic Activity Levels } \\
\hline \multirow[t]{3}{*}{ DF } & MGAS $\$$ & $0.08(0.06)$ & $0.09(0.07)$ & $0.09(0.06)$ & $0.13(0.10)$ & $0.13(0.08)$ & $0.18(0.11)$ & $0.19(0.23)$ & $0.19(0.12)$ \\
\hline & LGAS $^{*}$ & $0.13(0.07)$ & $0.15(0.12)$ & $0.15(0.08)$ & $0.21(0.14)$ & $0.23(0.16)$ & $0.28(0.15)$ & $0.25(0.15)$ & $0.48(0.56)$ \\
\hline & SOL & $0.20(0.14)$ & $0.23(0.15)$ & $0.20(0.13)$ & $0.29(0.21)$ & $0.26(0.18)$ & $0.32(0.22)$ & $0.27(0.18)$ & $0.38(0.28)$ \\
\hline PF & TAT & $0.20(0.14)$ & $0.09(0.04)$ & $0.22(0.14)$ & $0.12(0.09)$ & $0.22(0.16)$ & $0.13(0.07)$ & $0.26(0.16)$ & $0.21(0.14)$ \\
\hline
\end{tabular}

Standard deviations in parentheses. Normalization is to maximum isometric activity level.

$\ddagger$ Significant age $(P=.002)$ and velocity $(P<.001)$ effects by ANOVA.

$\$$ Significant velocity $(P<.001)$ effects by ANOVA.

+ Significant age $(P<.001)$ and velocity $(P<.008)$ effects by ANOVA.

- Significant age $(P=.008)$ and velocity $(P<.001)$ effects by ANOVA.

See Table 1 for definitions of abbreviations.

The columns headed 30,60 , and $120 \mathrm{deg} / \mathrm{s}$ give the isokinetic test results at those nominal angular velocities.

comfort and experimental convenience. Our concern was mostly with the timing of the MES, which likely was little affected by our choice of electrode type. As to MES magnitudes, each subject served as his own, and each muscle as its own, control in the sense that MES magnitudes were most often compared with maximum isometric exertion magnitudes for that electrode on that subject when that muscle acted as an agonist.

\section{ACKNOWLEDGMENTS}

The authors gratefully acknowledge the assistance of Murrie Green, Janet Grenier, Julie Grunawalt, and Rhonda Keller in this research.

\section{REFERENCES}

1. Thelen DG, Schultz AB, Alexander NB, Ashron-Miller JA. Effects of age on rapid ankle torque development. J Gerontol 1995, in press.

2. Brooks SV, Faulkner JA. Contractile properties of skeletal muscles from young adult and aged mice. J Physiol (Lond) 1988;404:71-82.

3. Fugl-Meyer AR, Mild KH, Hornsten J. Output of skeletal muscle contractions: A study of isokinetic plantar flexion in athletes. Acta Physiol Scand 1982;115:193-199.

4. Rothstein JM, Delitto A, Sinacore DR, Rose SJ. Electromyographic peak torque and power relationships during isokinetic movement. Phys Ther $1983 ; 63: 926-933$.

5. Gerdle B, Fugl-Meyer AR. Mechanical output and IEMG of isokinetic plantar flexion in 40- to 64-year-old subjects. Acta Physiol Scand 1985;124:201211.
6. Osternig LR, Hamill J, Corcos DM, Lander J. Electromyographic patterns accompanying isokinetic exercise under varying speed and sequencing conditions. Am J Phys Med 1984;63:289-297.

7. DeJong RN. The Neurologic Examination, 4th Ed. Hagerstown, MD Harper and Row, 1979.

8. Kottke FJ, Stillwell GK, Lehmann JF. Krusen's Handbook of Physical Medicine and Rehabilitation, 3rd Ed. Philadelphia: W.B. Saunders, 1982.

9. Alexander NB, Schultz AB, Warwick D. Rising from a chair: Effects of age and functional ability on performance biomechanics. J Gerontol 1991;46:M91-98.

10. Wolfson LI, Katzman R. The neurological examination at age 80. In: Katzman R, Terry R, eds. The Neurology of Aging. Philadelphia: F.A. Davis, 1983.

11. Deleted in proof.

12. Potvin AR, Tourtellotte WW, Syndulko K, Potvin J. Quantitative methods in assessment of neurologic function CRC Crit Rev Bioeng 1981;6:177-224.

13. Gottsdanker R. Age and simple reaction time. J Gerontol 1982;37:342-348.

14. Inglin B, Woollacott $\mathrm{M}$. Age-related changes in anticipatory postural adjustments associated with arm movements. J Gerontol 1988;43:M105-113.

15. Welford AT. Reaction time speed of performance and age. Ann N Y Acad Sci 1988;515:1-17.

16. Woolacott $\mathrm{M}$, Inglin B, Manchester $\mathrm{D}$. Response preparation and posture control: Neuromuscular changes in the older adult. Ann N Y Acad Sci 1988;515:42-53.

17. Manchester D, Woolacott M, Zederbauer-Hylton N, Marin O. Visual vestibular and somatosensory contributions to balance control in the older adult. J Gerontol 1989;44:M118-127.

18. Solomonow M, Baratta R, Zhou BH, D'Ambrosia R. Electromyogram coactivation patterns of the elbow antagonist muscles during slow isokinetic movement. Exp Neurol 1988;100:470-477. 